\title{
Simple and efficient LCAO basis sets for the diffuse states in carbon nanostructures
}

\author{
Nick R. Papior, ${ }^{1}$ Gaetano Calogero, ${ }^{1}$ and Mads Brandbyge ${ }^{1}$ \\ ${ }^{1}$ Dept. of Micro- and Nanotechnology, Technical University of Denmark, \\ Center for Nanostructured Graphene (CNG), Ørsteds Plads, \\ Bldg. 345E, DK-2800 Kongens Lyngby, Denmarl ${ }^{*}$
}

(Dated: November 8, 2018)

\begin{abstract}
We present a simple way to describe the lowest unoccupied diffuse states in carbon nanostructures in density functional theory (DFT) calculations using a minimal LCAO (linear combination of atomic orbitals) basis set. By comparing plane wave basis calculations, we show how these states can be captured by adding long-range orbitals to the standard LCAO basis sets for the extreme cases of planar $s p^{2}$ (graphene) and curved carbon $\left(\mathrm{C}_{60}\right)$. In particular, using Bessel functions with a long range as additional basis functions retain a minimal basis size. This provides a smaller and simpler atom-centered basis set compared to the standard pseudo-atomic orbitals (PAOs) with multiple polarization orbitals or by adding non-atom-centered states to the basis.

PACS numbers: 31.15.aq, 71.15.Mb, 31.15.E-, 31.50.Df, 71.15.Ap, 71.20.-b,
\end{abstract}

The bandstructure of graphene around the Fermi level is a textbook example of the tight-binding model using just a single $p_{z}$-orbital $(z \perp$ graphene) per carbon atom and nearest neighbour interaction[1]. Therefore it is not surprising that it can be reproduced quite well by a simple linear combination of atomic orbitals (LCAO) with a single orbital per valence state per carbon atom corresponding to one $s$ and three $p$ orbitals $(M=4)$. It is generally of great interest to keep the basis-set size $(M)$ as small and simple as possible to keep the computational cost down, and to enable calculations based on density functional theory (DFT) of larger systems, for example electronic transport calculations of graphene-based devices using non-equilibrium Greens functions 2, 3. An accurate description of all the occupied bands, comparable to the result of calculations based on plane-waves (PW) basis sets, calls for the use of a larger LCAO basis set f.ex. using $M=13$ (two sets of $s, p$ and one set of $d$ ) atom-centered basis functions based on the atomic orbitals. This size is generally believed to be a good compromise between accuracy and computational cost and is a standard choice in LCAO-DFT codes such as SIESTA [4, OpenMX [5], or FHI-aims[6]. However, as pointed out by Stewart [7, this choice yields a wrong description of the first unoccupied bands, which start about $3.25 \mathrm{eV}$ above the Fermi level and are parabolic around the Brillouin zone center, $\Gamma$. These bands correspond to diffuse states with long tails into the vacuum, and are the first in a quasi-continuum of free electron-like bands in a double Rydberg series of image-potential-like states 8 with even and odd mirror symmetry in the graphene plane. In particular, the first two unoccupied states $\left(1^{ \pm}\right)$are important for e.g. the description of interlayer states, reactivity, intercalation [9, 10], and tunneling into graphene, where the inelastic phonon scattering plays a dominant role 11, 12. States of similar origin has been found for the finite $\mathrm{C}_{60}$-molecule, representing another extreme compared to the flat, infinite graphene 13, 14. The diffuse molecular orbitals, dubbed Super Atom Molecular Orbitals (SAMOs) were observed in STM experiments [15, and are located $\sim 4 \mathrm{eV}$ above the Fermi level.

Here we propose a simple, long-ranged, atomiccentered basis set, which can capture the lowest unoccupied bands of graphene and the SAMO states of $\mathrm{C}_{60}$ in DFT-LCAO electronic structure calculations. Its construction is based on a straightforward extension of standard basis sets, and yield a level of accuracy comparable to $\mathrm{PW}$ calculations for the first two unoccupied, diffuse bands (states) for graphene $\left(\mathrm{C}_{60}\right)$.

Quantum chemists traditionally use Gaussian-type orbitals(GTO) as bases. Another approach is to use solutions to the free atoms, e.g. described by pseudo-potentials, and to confine these within maximum range 16]. These pseudo-atomic orbitals $(\mathrm{PAO})$ can be used as a LCAO basis - a so-called single- $\zeta$ (SZ) basis corresponding to $M=4$ for carbon. The basis set can be improved by splitting each $\mathrm{PAO}$ into a part representing the center and another part representing its tail, doubling the number of $\zeta$-functions describing each valence orbital (double- $\zeta$ or DZ) 17. To improve further one can add orbitals with higher angular momentum $(l)$ than present in the valence shell, which for carbon amounts to the $d$ shell, $l=2$. These additional basis functions are termed "polarization" and can be generated by applying a perturbing polarizing electric field to the free atom[17]. The double- $\zeta$ plus polarization (DZP) is thus amounting to $M=2 \times 4+5=13$ basis functions for carbon and comprise a standard LCAO basis set in SIESTA 4], which is the DFT-LCAO code we use in this study. However, one may use the splitting procedure 17 to generate more refined bases such as triple- $\zeta$ or double-polarization. Importantly, however these are all basis functions originating from an atomic problem and thus have a decay away from the atomic core controlled by the atomic potential. We will return to this point later, additionally our main discussion covers graphene while $\mathrm{C}_{60}$ SAMOs are detailed 


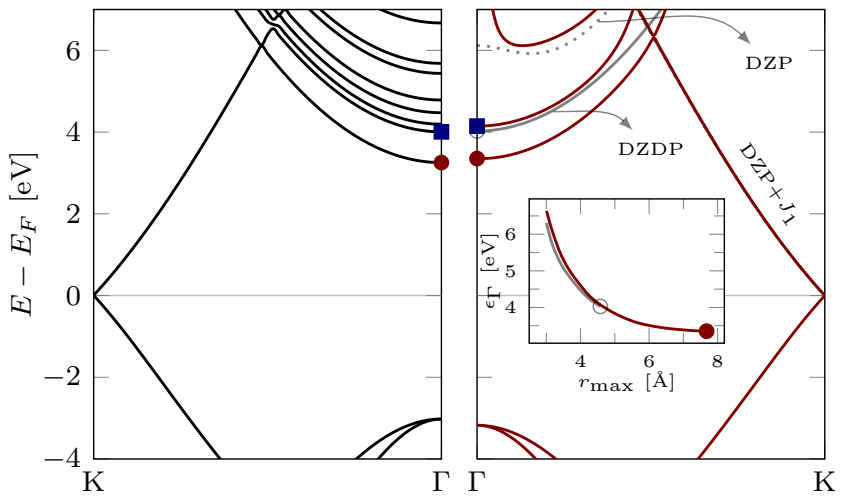

FIG. 1. Bandstructure of graphene along $\Gamma-\mathrm{K}$ obtained from a $\mathrm{PW}$ basis set (left) in comparison with the equivalent bandstructure from three different LCAO basis sets (right): a standard DZP (grey dotted) with an orbital-confining radii cutoff $\delta E=0.0025 \mathrm{eV}$, a DZDP (grey solid) with the same $\delta E$, and a DZP with $\delta E=0.1 \mathrm{eV}$ combined with two Bessel functions $J_{l \in\{0,1\}}$ and hard-wall potential range $r_{\max }=7.5 \AA$ (red solid). The eigenvalues at $\Gamma$ for the first $\left(1^{+}\right)$and second $\left(1^{-}\right)$ lowest unoccupied bands are marked by a red circle and a blue square, respectively. The insert shows the convergence of the $1^{+}$eigenvalue at $\Gamma$ as a function of the basis $\left(J_{l}\right)$ range for the DZDP $\left(\mathrm{DZP}+J_{1}\right)$ basis set. The maximum available DZDP range was constrained to $\sim 4.6 \AA$ because of limitations in the choice of $\delta E$.

in the end.

Let us consider the electronic bandstructure of graphene in Fig. 1, calculated using a PW basis set from VASP [18, (left) and the DFT-LCAO code Siesta[4, 19] (right) for a selected choice of LCAO basis sets. We have employed the PBE[20] functional for exchangecorrelation, $k$-point sampling of $42 \times 42(96 \times 96, \mathrm{LCAO})$, and a carbon-carbon distance of $a=1.42 \AA$. In the PW bandstructure the expected quasi-continuum of free electron-like vacuum states appears at the $\Gamma$-point above $3.25 \mathrm{eV}$. We focus on the first $\left(1^{+}\right)$and second $\left(1^{-}\right)$lowest unoccupied eigenstates, marked by a circle and square, respectively, and compare them to the LCAO bandstructures. We consider three different atomcentered bases, namely the standard DZP $(M=13)$, a double-polarization DZDP $(M=18)$ where the polarization $d$-orbitals are doubled, and a DZP basis extended by two Bessel functions $\left(J_{l}\right)$ with angular momentum $l \in\{0,1\}(M=13+4=17)$, in the following $J_{1}$ implicitly includes $J_{0}$ orbitals.

First we note that while all LCAO bases yield a good description of the occupied bands, the standard DZP basis set fails completely in reproducing the lowest unoccupied states, showing a non-parabolic $1^{+}$band around $\sim 5.8 \mathrm{eV}$ at $\Gamma$. The results suggests that the discrepancy is due to the limited DZP basis size, which cannot supply linear combinations to account for the free electron-like bands. As shown by Silkin et al. [8] the $1^{ \pm}$bands have $s$ and $p_{z}$ characters which are already the predominant part of the valence bands. Therefore the DZP basis can not account for both the free electron-like bands and the valence bands.

The easiest procedure towards correcting the shape and position of the lowest unoccupied band is to double the polarization orbitals, DZDP (or TZDP [10]). In this case it is the tail polarization $d$-orbitals that accounts for the missing linear combinations. Subsequent tuning of the range of the basis is necessary in order to obtain a better agreement with the PW results. This is done in the inset of Fig. 1 which shows the convergence of the DZDP (gray) lowest band at $\Gamma\left(\epsilon_{\Gamma}\right)$ with respect to the basis orbital cutoff radius.

We can obtain better and more economical results by using custom basis orbitals based on spherical Bessel functions 21. The Bessel functions are solutions to the spherical "particle-in-a-box" problem with hard-wall cutoff[22]. Importantly, these orbitals are not constrained by a core potential, and thus have a well defined shape depending only on the chosen radial cut-off and angular momentum $l$. An atomic orbital does not necessarily increase weight for large $r$ due to confinement potentials. Effectively this means that basis orbitals originating from atomic pseudopotentials tend to have a small cutoff radius regardless of user defined ranges. The first band can be described by a single long range $J_{0}$ Bessel function $(s)$, while the second band also requires $J_{1}(s+p)$. The $\Gamma-\mathrm{K}$ bandstructure in Fig. 1 (right) shows the $\mathrm{DZP}+J_{1}$ which is in good agreement with the PW calculation for the first two bands. An improved energy alignment with respect to the PW calculation can be achieved by extending the basis orbitals to as much as $r_{\max } \sim 7.5 \AA$ which was used above. The inset in Fig. 1 shows the convergence of the first band energy at the $\Gamma$-point for increasing $r_{\max }$ for the DZDP and DZP $+J_{1}$ basis sets.

Remark that $\mathrm{DZ}+J_{0} / \mathrm{DZ}+J_{1} / \mathrm{DZP}+J_{0} / \mathrm{DZP}+J_{1}$ all reproduce the first band with a band onset between $3.41 \mathrm{eV}$ and $3.35 \mathrm{eV}$, respectively. In Table $\mathrm{I}$ we list the two first unoccupied band-onsets at $\Gamma$ for the prominent DZ variants tested. All SZ variants yield $\epsilon_{1+}>5 \mathrm{eV}$, while the $\mathrm{TZ}$ variants are comparable to $\mathrm{DZ}$.

TABLE I. Positions of band energies at the $\Gamma$-point for the first $\left(1^{+}\right)$and second $\left(1^{-}\right)$unoccupied bands for different LCAO basis sets with size $M$ and maximal cut-off. PW shows the planewave benchmark calculation.

\begin{tabular}{c|ccccccc|c}
{$[\mathrm{eV}]$} & $\mathrm{DZ}$ & $\mathrm{DZ}+J_{0}$ & $\mathrm{DZ}+J_{1}$ & $\mathrm{DZP}$ & $\mathrm{DZP}+J_{0}$ & $\mathrm{DZP}+J_{1}$ & $\mathrm{DZDP}$ & $\mathrm{PW}$ \\
\hline$\epsilon_{1+}$ & 9.03 & 3.42 & 3.35 & 5.81 & 3.41 & 3.35 & 3.64 & 3.25 \\
$\epsilon_{1-}$ & 9.33 & 9.34 & 4.07 & 8.24 & 8.30 & 4.15 & 8.27 & 4.01 \\
$M$ & 8 & 9 & 12 & 13 & 14 & 17 & 18 & -
\end{tabular}

We note in passing that one could include the unoccupied band (only close to $\Gamma$ ) in the nearest neighbour tight-binding $p_{z}$-model[1] by adding an orbital with $s$ symmetry to each atom, thus yielding orbitals orthogo- 
nal to the $\pi$-system. The hopping parameter $\gamma^{+}$can be approximated by the regular $p_{z}$ hopping parameter since the two bands have nearly identical parabolic curvature close to $\Gamma$, effectively setting $\gamma^{+} \approx 2.7 \mathrm{eV}$ and on-site $\epsilon_{1+}+3 \gamma^{+}$. Further discussion of tight-binding models of the bands may be found elsewhere[23].

In Fig. $2 \mathrm{a}$ we compare the wavefunctions through a carbon atom along $z$ obtained by $\mathrm{PW}$ and LCAO, respectively. These also show a reasonable agreement with the PW results. Note how the LCAO tails are forced zero for $r>7.5 \AA$. The symmetric lowest state $1^{+}$(bottom) is accurately described by LCAO although the tail for PW extends farther into vacuum. The anti-symmetric second lowest state $1^{-}$(top) is more extended in PW compared to LCAO, as expected.

The density of states (DOS) is shown in Fig. $2 \mathrm{~b}$ comparing the $\mathrm{PW}$ calculation with the four selected basis sizes. $k$-point sampling was converged. A large improvement in the description of the unoccupied bands accompanies the appropriate choice of basis size. Clearly, $\mathrm{DZP}+J_{0} / J_{1}$ reproduce the DOS to a satisfactory level. The difference between PW and LCAO DOS shapes are mainly due to different smearing methods. In Fig. 2 c the projected DOS onto the basis functions for DZP $+J_{1}$ highlights how the unoccupied bands indeed are of $s$ $\left(1^{+}\right)$and $p$-character $\left(1^{-}\right)$. Thus the $1^{+}$-state consists of $s$ with a negative $p_{x(y)}$ where the band starts (at $\Gamma$ ), while the $1^{-}$-state has $p_{z}$ odd character symmetry.

Along similar lines Agapito and co-workers [10] considered projections of different LCAO basis-sets onto planewave Bloch states as well as DFT-LCAO calculations. They found that a TZDP $(M=22)$ basis set with a cutoff range of $4 \AA$ did not reproduce the $1^{ \pm}$-bands and had to use a DZP supplemented with long-ranged (cutoff $6.9 \AA$ ) empty-atom (EA) basis-functions located $2.8 \AA$ outside the graphene plane to get a reasonable description of these. Besides being costly to use a DZP $+\mathrm{EA}$ basis, it also makes calculations conceptually and practically more difficult for systems where one e.g. adsorb or bind molecules to graphene. A large overlap between the EA-basis and the adsorbates may lead to spurious effects.

As outlined above Bessel functions are advantageous in the graphene case. Another approach uses long range $3 \mathrm{~s}$ and $3 p$ carbon atomic orbitals, which also correctly describes the graphene unoccupied states and with equivalent precision and basis size $M$ as $J_{1}$. However, for SAMO states of the $\mathrm{C}_{60}$-molecule we could only reproduce the $s$-character SAMO using the Bessel basis (further fine tuning of $3 s / 3 p$ orbitals may be able to capture the SAMOs). In Figure 3 we show the wavefunctions of the $s$ and $p$ SAMOs (produced by $\mathrm{DZP}+J_{0}$ ) along with the DOS in the respective energy range, $\mathrm{PW}$ calculations using $(40 \AA)^{3}$ cell. The shape of the wavefunctions compare well with those obtained with PW calculations [15]. Comparing DOS shows that the $s$ SAMO for PW and $J_{0}$
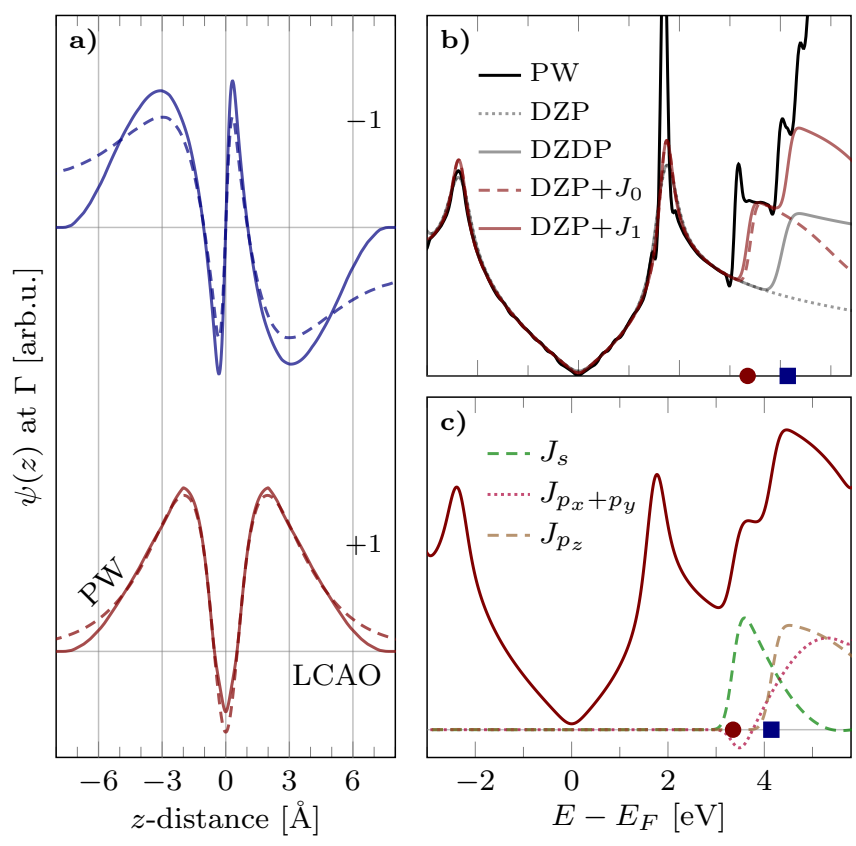

FIG. 2. (a) Comparison of normalized wavefunctions at $\Gamma$ obtained with PW basis (dashed), and LCAO with the DZP $+J_{1}$ basis (full). The wavefunctions at $\Gamma$ are projected on a line through a carbon atom. The $+1 /-1$ states are plotted in bottom/top parts, respectively. (b) Total DOS from PW in comparison with DZP, DZDP and DZP $+J_{0} / J_{1}$ basis sets. The onsets of $1^{ \pm}$at $\Gamma$ are highlighted on the energy axis. (c) Orbital resolved highest contributions to the DOS from DZP $+J_{1}$ in correspondence of the two lowest unoccupied bands.

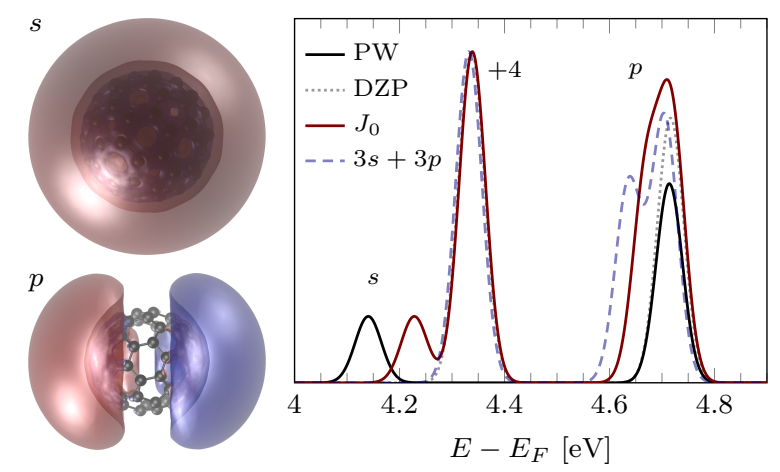

FIG. 3. Left: Iso-surface plots of the $s / p$ SAMO from LCAO $J_{0}$ calculation. Right: DOS comparison with PW, DZP, $J_{0}$ and $3 s+3 p$ basis, aligned at $E_{F}$. The LUMO+4 level is indicated as a reference to the rest of the $\mathrm{C}_{60}$-states. The $J_{0}$ basis reproduces the $s$ and $p$ SAMO while the much larger $3 s+3 p$ basis only reproduces the $p$ SAMO.

are separated by $\sim 0.1 \mathrm{eV}$, while the $3 s+3 p$ could not reproduce the $s$ state. Note that the PW LUMO+4 position is not in this energy range. These states are highly dependent on the cell (vacuum) size. The PW $s$ SAMO state is fixed in energy for $30 \AA$ and $40 \AA$ cell sizes.

In conclusion we have shown that the two lowest un- 
occupied diffuse states for graphene and $\mathrm{C}_{60}$ can adequately be described within the DFT-LCAO framework by adopting a conceptually and computationally simple atomic-centered basis set where Bessel functions with a long extension are supplementing the standard DZPbasis. The presented basis set provides a good compromise with respect to efficiency, due to the relatively small number of orbitals required, while ensuring a level of accuracy which is comparable to DFT calculations based on the planewave basis. The Bessel basis sets may be relevant in other $2 \mathrm{D}$ materials and/or surface calculations 24, 25]. The first two bands of graphene may be selected by choosing the symmetry of the basis function $\left(J_{0}\right.$ or $\left.J_{1}\right)$, while for $\mathrm{C}_{60} J_{0}$ is enough. Consequently only adding 1 basis orbital per atom to the DZ/DZP basis set will correctly describe the first unoccupied band of graphene.

We are grateful to Dr. Aran Garcia-Lekue and Mr. Bernhard Kretz for useful discussions. We acknowledge funding from Villum Fonden (grant no. 00013340), Danish research council (grant no. 4184-00030). The Center for Nanostructured Graphene (CNG) is sponsored by the Danish Research Foundation, Project DNRF103.

* nickpapior@gmail.com

[1] M. L. Cohen and S. G. Louie, Condensed Matter Physics, edited by NN (Cambridge University Press, 2016).

[2] N. Papior, T. Gunst, D. Stradi, and M. Brandbyge, Physical Chemistry Chemical Physics 18 (2015), $10.1039 / \mathrm{c} 5 \mathrm{cp} 04613 \mathrm{k}$.

[3] D. Stradi, N. Papior, O. Hansen, and M. Brandbyge, Nano Letters 17 (2017), 10.1021/acs.nanolett.7b00473.

[4] J. M. Soler, E. Artacho, J. D. Gale, A. García, J. Junquera, P. Ordejón, and D. Sánchez-Portal, Journal of Physics: Condensed Matter 14, 2745 (2002)

[5] T. Ozaki, Physical Review B 67, 155108 (2003).

[6] V. Blum, R. Gehrke, F. Hanke, P. Havu, V. Havu, X. Ren, K. Reuter, and M. Scheffler, Computer Physics
Communications 180, 2175 (2009)

[7] D. Stewart, Computing in Science \& Engineering 14, 55 (2012)

[8] V. M. Silkin, J. Zhao, F. Guinea, E. V. Chulkov, P. M. Echenique, and H. Petek, Physical Review B 80, 121408 (2009)

[9] M. Posternak, A. Baldereschi, A. J. Freeman, E. Wimmer, and M. Weinert, Physical Review Letters 50, 761 (1983)

[10] L. A. Agapito, M. Fornari, D. Ceresoli, A. Ferretti, S. Curtarolo, and M. B. Nardelli, Physical Review B 93 (2016), 10.1103/PhysRevB.93.125137

[11] Y. Zhang, V. W. Brar, F. Wang, C. Girit, Y. Yayon, M. Panlasigui, A. Zettl, and M. F. Crommie, Nature Physics 4, 627 (2008)

[12] T. O. Wehling, I. Grigorenko, A. I. Lichtenstein, and A. V. Balatsky, Physical Review Letters 101, 216803 (2008)

[13] M. Feng, J. Zhao, T. Huang, X. Zhu, and H. Petek, Accounts of Chemical Research 44, 360 (2011).

[14] S. Hu, J. Zhao, Y. Jin, J. Yang, H. Petek, and J. G. Hou, Nano Letters 10, 4830 (2010).

[15] M. Feng, J. Zhao, and H. Petek, Science 320, 359 (2008).

[16] O. F. Sankey and D. J. Niklewski, Physical Review B 40, 3979 (1989).

[17] E. Artacho, D. Sánchez-Portal, P. Ordejón, A. García, and J. M. Soler, physica status solidi (b) 215, 809 (1999)

[18] G. Kresse and D. Joubert, Phys. Rev. B 59, 1758 (1999).

[19] N. R. Papior, "sisl," Nww.github.com/zerothi/sisl (2018).

[20] J. P. Perdew, K. Burke, and M. Ernzerhof, Phys. Rev. Lett. 77, 3865 (1996)

[21] P. D. Haynes and M. C. Payne, Computer Physics Communications 102, 17 (1997)

[22] D. J. Griffiths, Introduction to Quantum Mechanics (Cambridge University Press, 2017).

[23] E. Kogan, V. U. Nazarov, V. M. Silkin, and M. Kaveh, Phys. Rev. B 89, 165430 (2014)

[24] C. Moreno, M. Vilas-Varela, B. Kretz, A. Garcia-Lekue, M. V. Costache, M. Paradinas, M. Panighel, G. Ceballos, S. O. Valenzuela, D. Peña, and A. Mugarza, Science 360, $199(2018)$

[25] S. García-Gil, A. García, N. Lorente, and P. Ordejón, Physical Review B 79, 075441 (2009) 